\title{
Nicotine poisoning increase due to e-cigarettes
}

$\mathrm{N}$ icotine-poisoning related to the use of e-cigarettes increased $300 \%$ last year in the US, but Canada's fragmented poison data-gathering system reveals only a disturbing lack of information.

In the United States, the American Association of Poison Control Centers advised the public to use caution with e-cigarette devices and highly concentrated liquid nicotine after a surge in accidental poisonings.

In 2013, there were 1414 reported exposures to e-cigarette liquids; a $300 \%$ increase over 2012. Of these reported cases in 2013, 365 people were referred to hospital, which is triple the number in 2012. As of Mar. 24, 2014, there were 651 reported exposures; more than half among children under age six.

Health Canada hasn't approved e-cigarettes, but has outsourced a contract to monitor retail sales. Although the products are available, information on nicotine poisoning is not.

"Canada does not have a national poisoning database and statistics are lacking," says Martin Laliberté, president of the Canadian Association for Poison Control Centre. "Exposures to nicotine from e-cigarettes have been reported by provincial poison centres, but it is currently impossible to establish if nicotine poisonings are increasing."

He acknowledges that the increased availability of e-cigarettes "make nicotine sulfate more available and an increase in nicotine exposures seems a

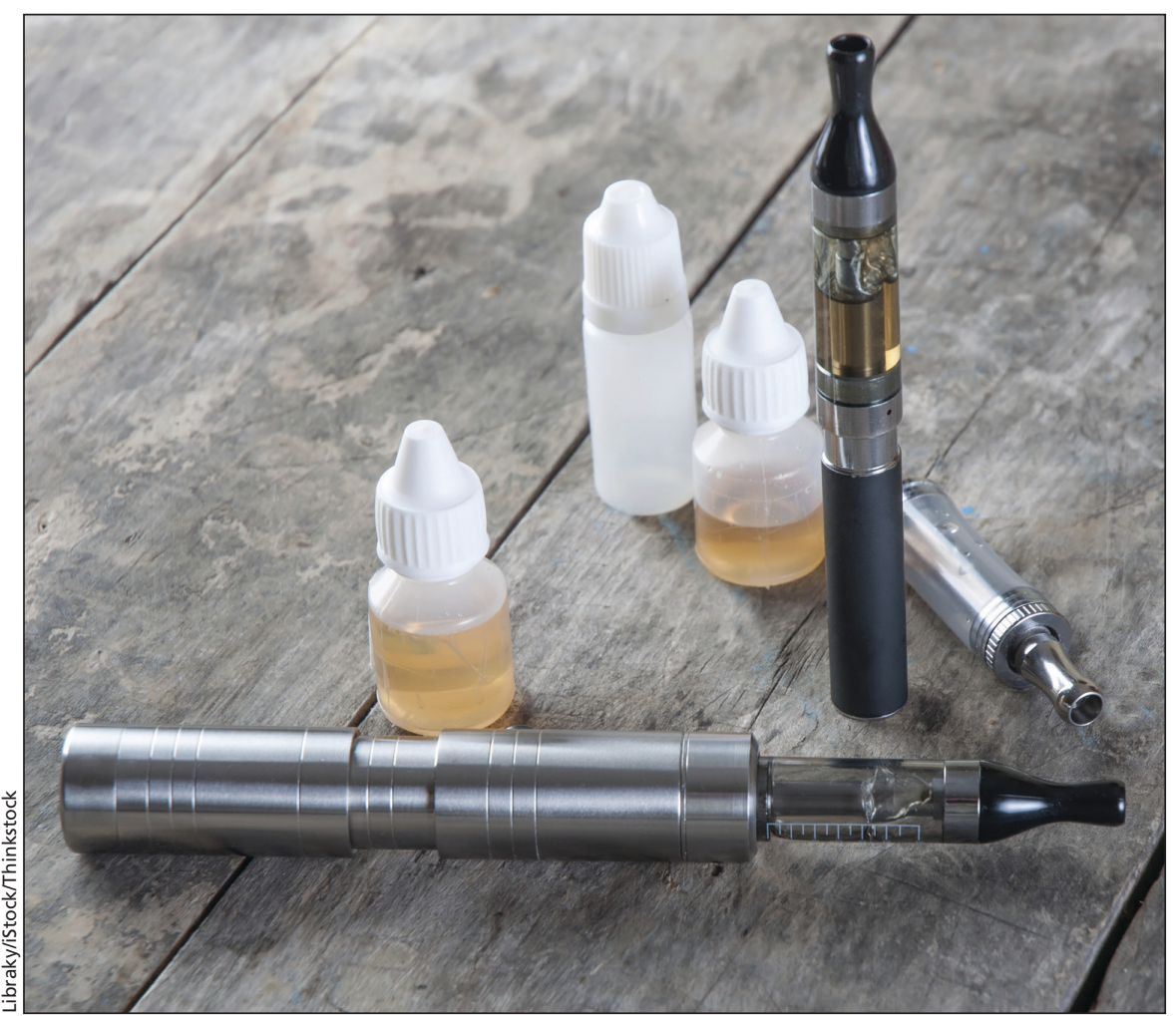

The US reports a $300 \%$ increase in accidental poisonings from the liquid nicotine sulfate used in e-cigarettes.

very likely consequence." He is also concerned that the flavoring of the nicotine liquids makes these products very attractive to children.

"This is a potential public health safety issue and many of us in the medical toxicology community are worried about the risk of exposure to nicotine sulfate in children and adults as well."

The liquid used in e-cigarettes contains nicotine sulfate, a very powerful neurotoxin, which can be absorbed through the skin or ingested. It was previously used as an insecticide, but was withdrawn in part because of the risk of toxicity associated with skin exposure.

"Perhaps e-cigarettes users should be reminded that vaporizing an insecticide is not trendy at all." - Adam Miller, Toronto, Ont.

CMAJ 2014. DOI:10.1503/cmaj.109-4818 